\title{
Translating Higher-Order Specifications to Coq Libraries Supporting Hybrid Proofs
}

\author{
Nada Habli ${ }^{1}$ and Amy P. Felty ${ }^{1,2}$ \\ 1 Department of Mathematics and Statistics, University of Ottawa, Canada \\ 2 School of Electrical Engineering and Computer Science, University of Ottawa, Canada \\ nhabl094@uottawa.ca, afelty@eecs.uottawa.ca
}

\begin{abstract}
We describe ongoing work on building an environment to support reasoning in proof assistants that represent formal systems using higher-order abstract syntax (HOAS). We use a simple and general specification language whose syntax supports HOAS. Using this language, we can encode the syntax and inference rules of a variety of formal systems, such as programming languages and logics. We describe our tool, implemented in OCaml, which parses this syntax, and translates it to a Coq library that includes definitions and hints for aiding automated proof in the Hybrid system. Hybrid itself is implemented in Coq, and designed specifically to reason about such formal systems. Given an input specification, the library that is automatically generated by our tool imports the general Hybrid library and adds definitions and hints for aiding automated proof in Hybrid about the specific programming language or logic defined in the specification. This work is part of a larger project to compare reasoning in systems supporting HOAS. Our current work focuses on Hybrid, Abella, Twelf, and Beluga, and the specification language is designed to be general enough to allow the automatic generation of libraries for all of these systems from a single specification.
\end{abstract}

\section{Introduction}

The Hybrid system 4 provides support for reasoning about object languages (OLs) such as programming languages and other formal systems using higher-order abstract syntax (HOAS). In [4, two versions of Hybrid are described, one implemented in Isabelle [8], and one implemented later in the Coq Proof Assistant [1] by fairly directly porting the Isabelle version to Coq. We focus on the Coq version here. Hybrid provides support for encoding syntax, for representing the semantics via inference rules and axioms, and for reasoning about the properties of the OL. For example, reasoning about the metatheory of a programming language allows important properties, such as soundness, to be established formally. Such properties are important for providing assurance that a language can be used to build reliable and secure software systems.

The general Hybrid infrastructure is implemented as two Coq libraries. The first provides an underlying de Bruijn representation of $\lambda$-terms parameterized by a set of constants for a particular OL. This layer is hidden from the user. This library includes a set of definitions and lemmas that builds an HOAS layer from this lower level, which is used to encode the syntax of OLs. The only axiom used in the implementation is the law of excluded middle, included by importing Coq's library for classical logic. ${ }^{1}$ The reasoning infrastructure has multiple levels also. The inference rules of an OL are defined at the lowest level as logic programming-like clauses (called prog clauses here) that are provided as a parameter to an intermediate logic,

\footnotetext{
${ }^{1}$ This library was originally imported in order to keep the Coq implementation close to the Isabelle one. It is in fact not necessary. See [2] for a constructive version of Hybrid in Coq.
} 
called a specification logic (SL). The second Coq library implements the SL. At the highest level is the reasoning logic, which is Coq.

This paper presents our tool for supporting reasoning in Hybrid by translating high-level specifications of OLs to Coq libraries. Our specification language has three sections, Syntax, Judgments, and Rules. The first section contains the specification of new constants and their types, representing the basic syntax constructors of the OL. The allowed types are a subset of the types of the simply-typed $\lambda$-calculus. In HOAS, object-level binding is encoded directly using meta-level binding, and thus arguments to constructors are allowed to have function types. We restrict to second-order types, which means that the functions appearing as arguments must themselves take arguments of atomic types. Hybrid itself is currently restricted to second-order since the representation of many formal systems does not require more. The declarations in this section are used to generate the set of constants needed for the de Bruijn level, as well as a set of definitions for encoding syntax at the HOAS level.

The declarations in the second section introduce SL-level predicates. These are the predicates used to encode the judgments in the inference rules defining the semantics of the OL. The third section defines the OL inference rules, which are translated to prog clauses. Together, the predicates and clauses instantiate the required parameters of the SL.

We present the specification language and our translation tool informally via an example, which is described in Section 2. In Section 3, we describe the technical details of some of our algorithms and their implementation. This work is part of an ongoing larger project to compare reasoning in a variety of systems that reason using HOAS (see [3], for example). In Section 4 , we discuss the current focus of our work on the translation tool in the context of this larger project. In Section 5, we conclude and discuss our longer term goals.

\section{An Example: The Polymorphic $\lambda$-Calculus}

As an example, we consider typing for the polymorphic $\lambda$-calculus as defined in 10. The syntax is defined by the following grammars, and typing is defined by the rules below.

$$
\begin{aligned}
\text { Terms } \quad M, N \quad:= & x|\lambda x: T . M| M M|\lambda \alpha . M| M[T] \\
\text { Types } \quad S, T \quad::= & \alpha|T \rightarrow T| \forall \alpha . T \\
\frac{x: T \in \Gamma}{\Gamma \vdash x: T} t y_{v} & \frac{\Gamma \vdash M: S \rightarrow T}{\Gamma \vdash M N: T} \quad \Gamma \vdash N: S \\
& \frac{\Gamma \vdash M: \forall \alpha . T}{\Gamma \vdash M[S]:[S / \alpha] T} t y_{a} \quad \frac{\Gamma, x: S \vdash M: T}{\Gamma \vdash \lambda x: S . M: S \rightarrow T} t y_{l} \\
& \frac{\Gamma, \alpha \vdash M: T}{\Gamma \vdash \lambda \alpha . M: \forall \alpha . T} t y_{t l}
\end{aligned}
$$

Figure 1 encodes the syntax and typing rules, and illustrates the use of our specification language. In the Syntax section, the keyword type introduces new atomic types for the different syntax classes, which are the polymorphic types (tp) and terms (tm) in this example. Abstraction in types and terms (defined by constants all, lam, and tlam) is defined using function types. Thus in the HOAS representation, abstraction in the OL will be represented using abstraction in the meta-language, which here is Coq's $\lambda$-abstraction.

The typing judgment for the polymorphic $\lambda$-calculus is expressed using the typeof predicate declared in the Judgments section. Here, the keyword type will map to the type of propositions of the target system, which for Hybrid is the type of formulas of the SL. Note that we use the 


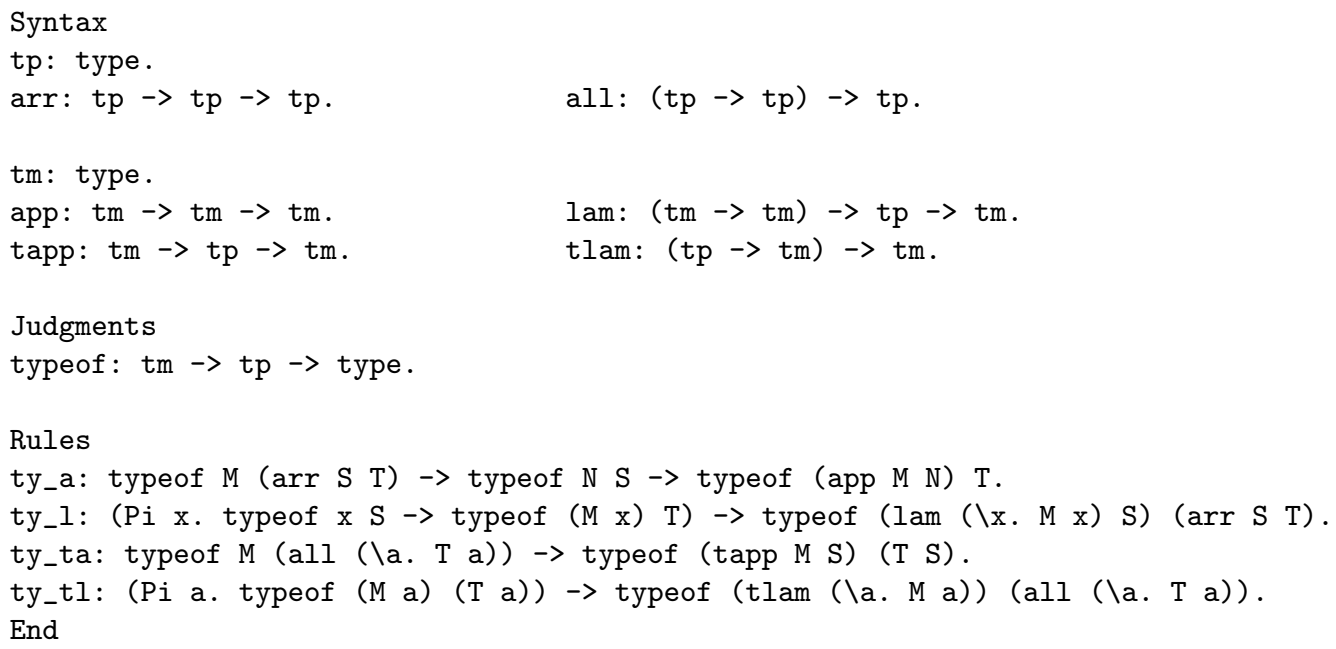

Figure 1: A Specification for the Polymorphic $\lambda$-Calculus

type keyword in both sections, borrowing from Twelf [11, where predicates and types are at the same level.

The inference rules appear in the last section, and here again the syntax resembles the syntax of Twelf to some extent. As in Twelf, the contexts that are explicit in the informal presentation of the rules are implicit in the judgment section of the specification. The rules are named, and the arrow is used to separate hypotheses from one another and from the conclusion, which is the last formula before the terminating dot. Binders in the polymorphic $\lambda$-calculus are represented using the binding operator of our specification language (backslash). We use tokens starting with uppercase letters for "schematic" variables (used to represent terms and types of the $\lambda$ calculus in this example) and tokens starting with lowercase letters for constructors, predicates, rule names, and bound variables.

From this fairly small specification, we generate a library that can be directly loaded into Coq, part of which is shown in Figures 2 and 3 As mentioned earlier, Hybrid is implemented in both Isabelle and Coq, and both implementations are described in 4. As we present the Coq code in this section, we will often refer to results from [4] that are relevant. For the reader interested in looking up these results, we note that most of the formal definitions and statements in that paper use a pretty-printed version of code that can be viewed as either Isabelle or Coq syntax. (See pages 48-49 for a description of this notation.) In the text of [4, when the implementations diverge, it is explicitly stated. (For example, see Section 2.2.)

The set Econ in Figure 2 is the set of constants that serve as a parameter to the de Bruijn representation of terms. Note that there is one for each constructor in the Syntax section. The next 3 lines perform this parameter instantiation and are the same for any Hybrid OL library. The last 6 lines of the Constants section fill in the Hybrid definitions for the HOAS representation of the 6 constructors. They are defined in terms of their underlying de Bruijn representation. The constant lambda is a binding operator defined on top of the de Bruijn representation, and its definition is part of the infrastructure hidden from the user. Types of bound variables in Coq are not explicitly added since they can be inferred. In these Coq definitions, there is no distinction between the types tp and $\mathrm{tm}$ found in the specification. All terms have Coq type uexp (the type of de Bruijn terms parameterized by the set ECon) and all 


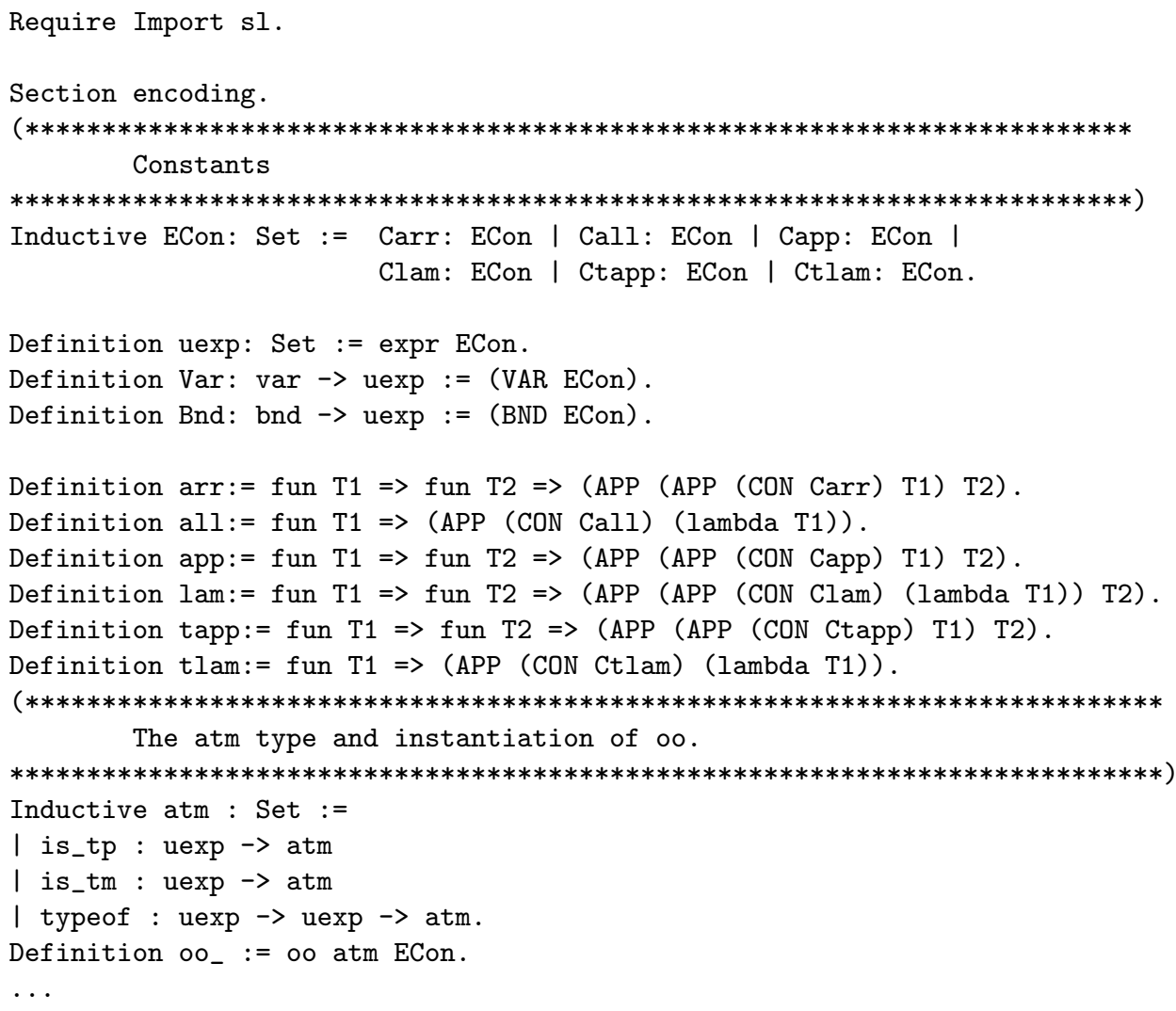

Figure 2: A Hybrid Library for Reasoning about the Polymorphic $\lambda$-Calculus Part 1

arguments to lambda have type (uexp $\rightarrow$ uexp).

Note that not all Coq functions of type (uexp $\rightarrow$ uexp) encode object-level $\lambda$-terms. Those that do not are often called exotic terms. Only functions that behave uniformly or parametrically on their arguments represent $\lambda$-terms. Hybrid includes a predicate abstr that rules out exotic terms and identifies exactly those terms that represent OL terms. (See Section 2, pages 52-54 in [4] for a definition of abstr as well as other definitions it depends on.) This predicate appears in the Coq code obtained from translating the OL inference rules to prog clauses. (See Figure 3.)

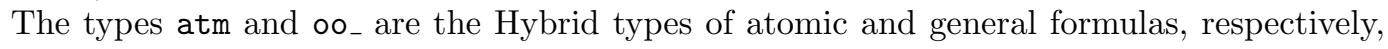
of the SL. A sequent calculus for the SL is implemented in Hybrid as an inductive predicate defining the type oo.. In 4, two sample SLs are given, one for a fragment of second-order intuitionistic logic, and another for an ordered linear logic. The former is used in the work described here. (See Figure 5 on page 68 of [4.) The sequent calculus is analogous to a logic programming interpreter, where the prog clauses can be viewed as a second-order logic program. We note that contexts in the SL are explicitly represented in the inductive definition, while they are implicit in the prog clauses. For readers familiar with Twelf, the prog clauses correspond to a Twelf program, while the SL corresponds to Twelf's meta-level, where OL contexts are represented as meta-level contexts. In Hybrid, both levels are formalized, and thus contexts are explicitly represented and reasoned about at the SL level. 


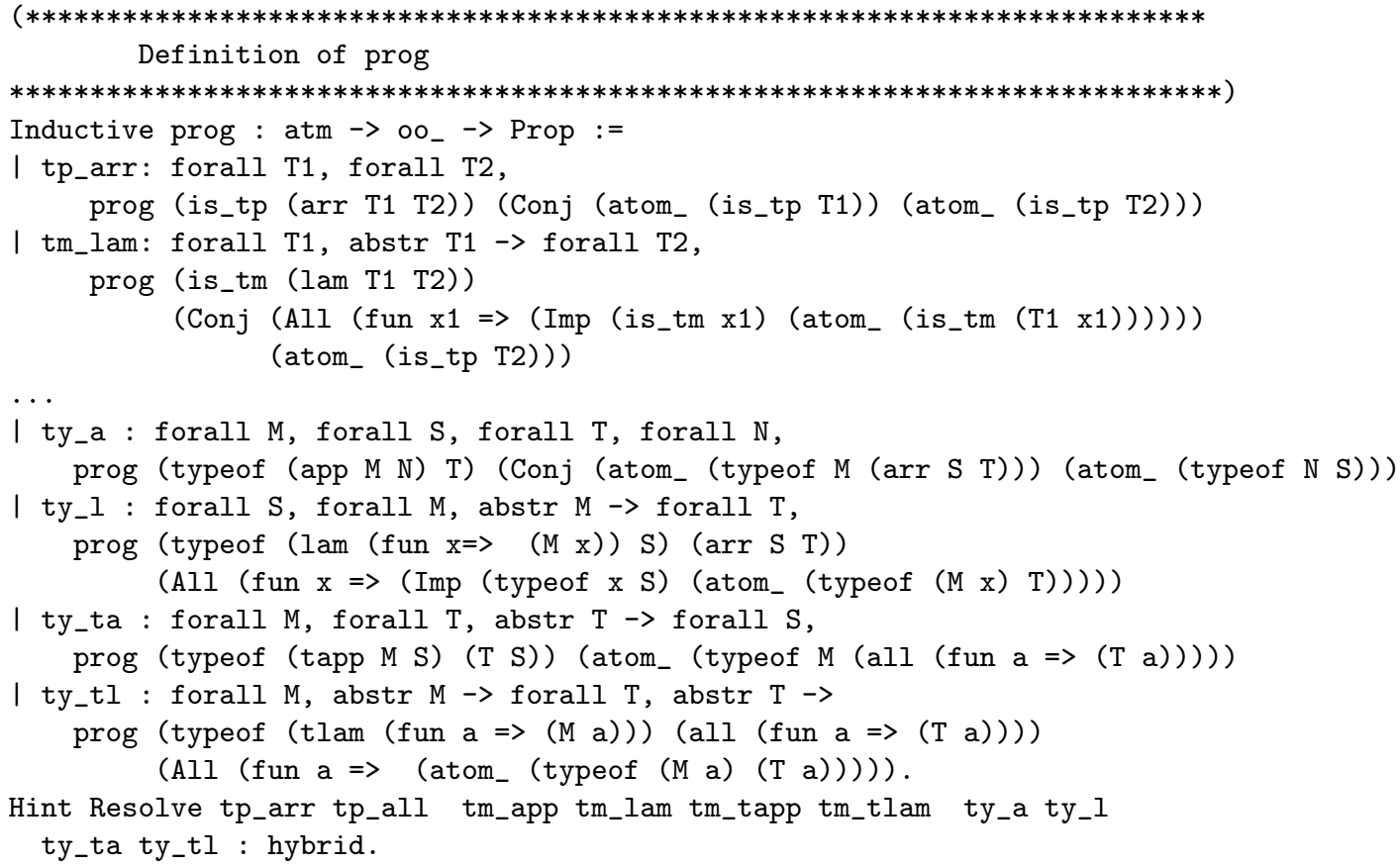

Figure 3: A Hybrid Library for Reasoning about the Polymorphic $\lambda$-Calculus Part 2

Figure 2 defines atm as an inductive set of predicates. Continuing the logic programming analogy, these can be viewed as predicates of a logic program. The binary predicate typeof comes directly from the specification. Again, the types of the arguments of this predicate (tm and $t p$ ) in the specification are mapped to uexp in the Coq library. As a result, we need to introduce a predicate corresponding to each type in the specification to be used to identify wellformed types and terms of the polymorphic $\lambda$-calculus. Here, the is_tp and is_tm predicates are introduced for this purpose. The last definition in the figure instantiates the oo - type, which must be done after the atm parameter is defined. The elided part includes other definitions involved in instantiating this type, as well as hints to Coq to help with automating proofs.

Figure 3 defines the prog clauses (or logic program) which serve as the final parameter to the SL. The last 4 clauses are direct translations of the rules in the specification. The prog predicate takes two arguments: the conclusion of an inference rule (the head of a logic programming clause) followed by the premise or premises (the body of the logic programming clause). The constructors Conj, Imp, and All are the connectives of the SL, and atom_coerces atm to $0_{-}$. $\mathrm{Pi}$ in the specification language maps to All, embedded implication maps to Imp, and multiple hypotheses are separated by Conj. Note that schematic variables in the specification are implicitly quantified at the outermost level. Explicit quantifiers (forall in Coq) are added to each clause of the definition of prog as part of the translation.

The other clauses, including the elided ones, are the rules for determining well-formed terms and well-formed types. These are automatically generated from the type declarations in the Syntax section, and represent the most complex part of the translation implemented so far.

In Hybrid, as in other logical frameworks such as Twelf, we must be sure that the syntax and inference rules are adequately encoded in the meta-language. Proving adequacy involves proving 
that there is a one-to-one correspondence between the syntax of the OL and its representation in the meta-language, and that an OL judgment has a proof using the inference rules if and only if the encoded version of the judgment is provable in the logical framework. For an example of how adequacy is proved in Hybrid, see Section 3.2 of [4. The rules for well-formed terms of the OL are an important component of adequacy proofs in Hybrid. In our example OL, for instance, we must prove that whenever there is a proof in Hybrid that a term of the polymorphic $\lambda$-calculus has a particular type, then both the term and the type are well-formed (as defined by the clauses for is_tp and is_tm).

\section{Implementation of the Translation Tool}

In this section, we describe the overall structure of the implementation of the translation, which as mentioned, is in OCaml. We have not given a formal definition of the syntax of the specification language, so this description is informal. Using mllex and mlyacc, the 3 sections of a specification are each parsed to a list of type (string * exp) list. In each pair in the list, the first argument is the constructor, predicate, or rule name, and the second argument is an element of the following type:

type exp $=$ Type | Id of string | Arrow of exp * exp | App of string * exp list |

Lambda of string $* \exp * \exp \mid \mathrm{Pi}$ of string $* \exp * \exp$

There is a direct mapping of each operator in the specification to a constructor of exp. For example, the combination of $\backslash$ and the dot separating the bound variable from the term maps to Lambda. The type keyword maps to Type, and all other identifiers to Id. We unify the syntax of all three sections of the specification, even though the first two do not use Lambda and Pi. The translation function has the following overall structure, divided here into 4 steps, with details of step 3(e) filled in a bit further in Figure 4.

1. Parse the input file into three lists of declarations: $d s 1, d s 2$, and $d s 3$ where each one is the parsed input of Syntax, Judgments, and Rules, respectively.

2. Call functions to isolate the following variables:

(a) Typelist: from ds1, obtain the list of identifiers (strings) from declarations of the form "id:type." in the specification.

(b) Syntax_listName_aux: from ds1 and Typelist, obtain a list of lists of the remaining identifiers (OL syntax constructor names); use Typelist to group them into sublists according to the target types of the constructors (the types just before the terminating dots).

(c) Syntax_listExpr_aux: from ds1 and Typelist, form the list of lists of types of the constructors (expressed as elements of type exp), using the same groupings into sublists as above in (b).

(d) Rules_listName: from ds3, get the list of the identifiers corresponding to rule names.

3. Call functions to create the following strings using the variables from step 2.

(a) string1: from Syntax_listName_aux construct the string for the inductive definition of ECon, with one case of the Coq definition for each constructor in Syntax_listName_aux with names prepended by C. (See ECon in Figure 2) 
(b) string2: from Syntax_listName_aux and Syntax_listExpr_aux, construct a string with one line for each constructor, containing a Coq definition for the encoding of syntax for that constructor. Two cases must be considered, depending on whether the constructor's type is first- or second-order. If there is a functional argument, the lambda operator is used. (See the 6 definitions at the end of the Constants section of Figure 2, )

(c) string3: from Typelist construct a string for the inductive definition of atm containing all the clauses for the well-formedness predicates (those of type uexp $\rightarrow$ atm, see Figure 2.

(d) string4: from ds2 construct a string containing clauses of the inductive definition of atm, one for each predicate in the Judgments section. Judgments cannot have function arguments; their types are first-order. We simply count the number of argument types and write "uexp ->" for each one, ending the clause with atm. (See the last clause of the definition of atm in Figure 2, )

(e) string5: from Syntax_listName_aux and Syntax_listExpr_aux, construct a string containing all the prog clauses for well-formedness of OL terms. See Figure 4 for some details of the implementation. (See also Figure 3 , which contains 2 of 6 such clauses, with the rest elided.)

(f) string6: from ds3 construct a string containing one prog clause corresponding to each rule in the Rules section. We omit the details. (See the last 4 prog clauses in Figure 3.)

(g) string7: From Syntax_listName_aux and Rules_listName it is straightforward to construct the Hint string. (See the last line of Figure 3 )

4. Write the following strings to the output file in the appropriate order: strings representing Coq comments, fixed strings (library elements that are the same for all specifications), and the strings obtained from step 3 .

\section{Extensions}

In this section, we discuss the extensions of our tool that we are currently working on, as well as some other near-term goals.

There are a variety of standard lemmas that are useful for reasoning about OLs that can be directly generated from the specification. The next step in our current work is to add capabilities to our tool to automatically generate the statements of these lemmas from the specification. In addition, their proofs are mostly easily automated. Part of our work involves improving Hybrid to include better tactics for automating such proofs. In addition, we envision augmenting the translation to automatically insert parts of a proof script into the Coq libraries. Our current work involves studying the most effective way to combine these two techniques for automating proofs. This approach is used in a variety of other tools such as Krakatoa [6], which automatically generates Coq libraries for Hoare-style reasoning about correctness of Java programs, and uses tactics designed specifically for automating proofs in this domain.

We also have done some preliminary work on extending the specification language to include a declaration section for contexts, used to represent a set of hypotheses. Many theorems require reasoning about contexts, and our previous work on comparing systems [3] focused particularly on this aspect.

Examples of the kinds of "standard lemmas" that we would like to generate and prove partially or fully automatically include lemmas for adequacy and lemmas for dealing with 
Loop 1 (outermost): For each type name tname in Typelist, each corresponding list of constructors cnames in Syntax_listName_aux and list of expressions representing types ctypes in Syntax_listExpr_aux, execute Loop 2. Using the first 3 declarations in Figure 1 as an example, the following data is used the first time through Loop 2:

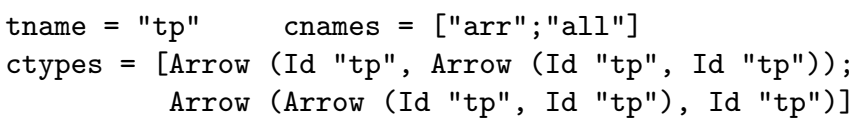

Loop 2 1. From tname and cnames, build a list rnames of rule names for prog clauses. (In the example, the result is ["tp_arr";"tp_all"].)

2. For each element rname of rnames and the corresponding element ctype of ctypes, execute Loop 3.

Loop 3 1. Count the number of arguments in ctype by finding the number of external arrows (all those except arrows in function types of arguments). Create a list args of pairs containing variables $\mathrm{T} 1, \mathrm{~T} 2, \ldots, \mathrm{Tn}$, one for each argument and arity of the argument ( 0 for non-functional arguments). For the first elements of ctypes, we get $[(" \mathrm{~T} 1 ", 0) ;(" \mathrm{~T} 2 ", 0)]$ and for the second element, we get $[(" \mathrm{~T} 1 ", 1)]$.

2. Using rname, ctype, and the corresponding element of args, build a string by concatenating the following substrings:

- "| " - rname - ":"

- For each ("Ti",m) in args, add "forall Ti,". If m > add "abstr Ti ->".

- "prog (is_" - tname - "(" - cname

- For each pair in args write the first element followed by a space. At the end, add "))".

- Create a string of the form ( $\left.\operatorname{Conj} s_{1}\left(\operatorname{Conj} s_{2} \ldots\left(\operatorname{Conj} s_{n-1} s_{n}\right) \ldots\right)\right)$ where $n$ is the number of elements of args. If $n=1$, the string is just $s_{1}$ with no Conj.

- If the $i^{\text {th }}$ element of args is ("Ti",0), $s_{i}$ is "(atom_ (is_" - t - " Ti))" where $t$ is the corresponding identifier in ctype (always "tp" in this example).

- If the $i^{\text {th }}$ element of args is ("Ti", m) where $\mathrm{m}>0$, then create variables $\mathrm{x} 1, \ldots, \mathrm{xm}$. Form $s_{i}$ as follows: for each $\mathrm{xj}$, add the substring "(All (fun $x j \Rightarrow$ (Imp (is_" - tj "xj)"; end $s_{i}$ with "(atom_ (is_" - $t_{-}$" $\left.\left.(\mathrm{Ti} \mathrm{x} 1 \ldots \mathrm{xm}) \ldots\right)\right) "$ where $\mathrm{tj}$ and $\mathrm{t}$ are the appropriate types in ctype.

For our example, from tname, the first elements of cnames and ctypes, and the first list args above, the output string we obtain is the first clause of the inductive definition of prog in Figure 3

Figure 4: Building string5 from Step 3(e).

explicit contexts, as well as a variety of others. For example, the adequacy lemma mentioned in Section 2 can be automatically generated. Many proofs in Hybrid proceed by induction over the SL with inversion over both the definitions of the SL and the prog clauses of the OL. In addition to the inversion lemmas automatically generated from a Coq inductive definition, we state and prove specialized inversion lemmas that can greatly simplify Hybrid proofs. The first lemma in Figure 5 is an example of such a lemma, one whose statement and proof can be automatically generated. Note that seq- is Hybrid's predicate for SL sequents. It takes 3 arguments: the height of a proof, a context of assumptions, and the formula to be proved. The proper predicate appearing in the lemma is important for adequacy (see 4]).

Context weakening is a general lemma that follows from the definition of the SL, and it 


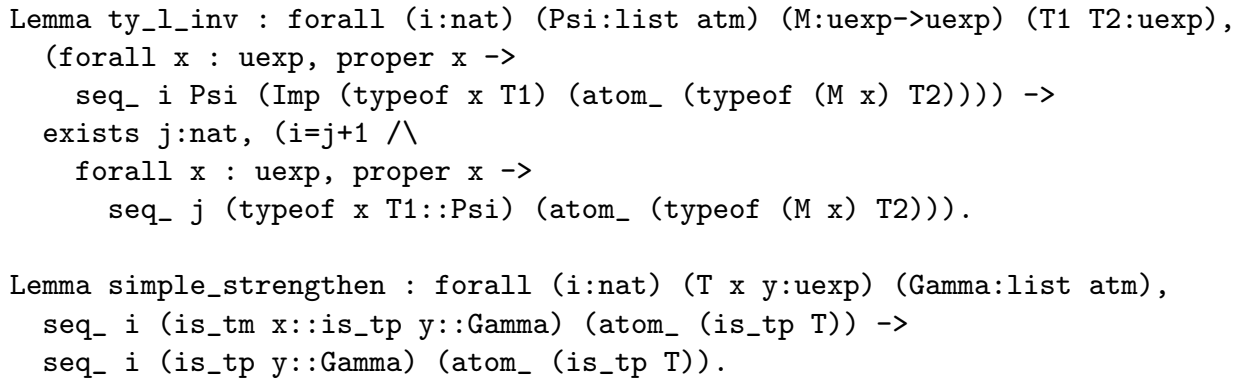

Figure 5: Example OL Lemmas

is stated and proved in the SL library. Context "strengthening" on the other hand, where assumptions that are irrelevant to the proof of a particular judgment are removed, depends on the OL. A simple example of a strengthening lemma is given in Figure 5 For several examples that occur in the context of a case study, see [3] (e.g., the occurrence of strengthening in the proof of Theorem 2). Part of our extension to the specification language will include the capability to specify at a high level what kinds of strengthening lemmas are desired, and then automatically generate and prove or partially prove them as part of the translation.

Our specifications can be translated to libraries for other systems supporting reasoning with HOAS, although we have not yet done so. Much of the work done here, however, can be directly reused, including, of course, the parsing of a specification to its internal representation in OCaml, as well as much of the overall structure of the OCaml functions we have defined to perform the translation. The systems we are currently targeting are Abella [5], Twelf [11, and Beluga [9]. A common characteristic of all of these systems is multi-level reasoning. A straightforward modification of the translation is all that should be required to obtain a basic input library for each of these systems. We mentioned earlier that our specification language adopts several features of Twelf directly. In fact, translation to Twelf will be the most straightforward to implement. Adding more significant support for each of these systems, such as including specialized lemmas, will require further effort.

\section{Conclusion and Future Work}

We have described our translation tool, which provides support for reasoning in Hybrid about object languages expressed using HOAS. We presented the specification language, described the translation to a Hybrid library, and discussed the implementation as well as several extensions planned for the near term.

In the longer term, we would also like to examine translations to more systems, in order to facilitate a more fuller comparison of reasoning in systems supporting HOAS. Such work may require extending the specification language. The work presented here can be considered as a variant of the Ott project 12 tailored specifically to the needs of HOAS. Ott contains constructs for specifying binders, and one of our longer term goals is to integrate our tool with Ott. In the present work, we chose to start with a smaller simpler language targeted to the needs of Hybrid, Abella, Twelf, and Beluga. Another approach is to consider using the higherorder logic programming language $\lambda$ Prolog [7] as both the specification language as well as the implementation language for the translation. 
Finally, we mentioned in Section 1 that both Hybrid and the specification language restrict the definition of syntax of OLs to second-order types. Another long-term goal is to generalize Hybrid to higher-order, which will then require extending our specification language and translation.

\section{References}

[1] Yves Bertot and Pierre Castéran. Interactive Theorem Proving and Program Development. Coq'Art: The Calculus of Inductive Constructions. Springer, 2004.

[2] Venanzio Capretta and Amy P. Felty. Combining de Bruijn indices and higher-order abstract syntax in Coq. In TYPES, pages 63-77, 2006.

[3] Amy Felty and Brigitte Pientka. Reasoning with higher-order abstract syntax and contexts: A comparison. In International Conference on Interactive Theorem Proving, volume 6172 of Lecture Notes in Computer Science, pages 227-242. Springer, 2010.

[4] Amy P. Felty and Alberto Momigliano. Hybrid: A definitional two-level approach to reasoning with higher-order abstract syntax. Journal of Automated Reasoning, 48(1):43-105, 2012.

[5] Andrew Gacek. The Abella interactive theorem prover (system description). In 4th International Joint Conference on Automated Reasoning, volume 5195 of Lecture Notes in Computer Science, pages 154-161. Springer, 2008.

[6] Claude Marché, Christine Paulin-Mohring, and Xavier Urbain. The Krakatoa Tool for Certification of Java/JavaCard Programs annotated in JML. Journal of Logic and Algebraic Programming, 58(1-2):86-106, 2004.

[7] Dale Miller and Gopalan Nadathur. Programming with Higher-Order Logic. Cambridge University Press, 2012.

[8] Tobias Nipkow, Lawrence C. Paulson, and Markus Wenzel. Isabelle/HOL: A Proof Assistant for Higher-Order Logic, volume 2283 of Lecture Notes in Computer Science. Springer Verlag, 2002.

[9] Brigitte Pientka and Joshua Dunfield. Beluga: A framework for programming and reasoning with deductive systems (system description). In 5th International Joint Conference on Automated Reasoning, volume 6173 of Lecture Notes in Computer Science, pages 15-21. Springer, 2010.

[10] Benjamin C. Pierce. Types and Programming Languages. MIT Press, 2002.

[11] Carsten Schürmann. The Twelf proof assistant. In 22nd International Conference on Theorem Proving in Higher Order Logics (TPHOLs), volume 5674 of Lecture Notes in Computer Science, pages 79-83. Springer, 2009.

[12] Peter Sewell, Francesco Zappa Nardelli, Scott Owens, Gilles Peskine, Thomas Ridge, Susmit Sarkar, and Rok Strniša. Ott: Effective tool suppor for the working semanticist. Journal of Functional Programming, 20(1):71-122, 2010. 